\title{
Naturalness of Artificial Intelligence ${ }^{\star}$
}

\author{
Jan Romportl ${ }^{1,2}$ \\ ${ }^{1}$ Department of Interdisciplinary Activities, New Technologies Research Centre \\ University of West Bohemia, Pilsen, Czech Republic \\ ${ }^{2}$ Department of Cybernetics, Faculty of Applied Sciences \\ University of West Bohemia, Pilsen, Czech Republic \\ rompi@kky.zcu.cz
}

\begin{abstract}
This position paper offers an answer to the question about the difference between artificial and natural. By building up a dichotomy between physis and logos, it argues that this difference is given by language and by what can be grasped with words. It concludes with an assertion that Good Old-Fashioned AI (GOFAI) cannot create anything natural, whereas emergent AI can, because emergent phenomena are intrinsically natural, which is a very important fact for the AI field. The paper also offers a view on the difference between the roles of an AI engineer in GOFAI and in emergent AI.
\end{abstract}

Keywords: artificial, natural, intelligence, logos, physis, language, horizon, emergentism, GOFAI.

\section{Introduction}

Many AI lectures and textbooks start by discussing what artificial intelligence means. It is quite convenient - especially when introducing the field to young and keen students of engineering - to dismiss the problem by saying that AI is "the science and engineering of making intelligent machines" ${ }^{1}$ and then continue with something "more useful", such as machine learning, artificial neural networks, pattern recognition, and so on.

A slightly different situation arises when AI is introduced to students of humanities, especially philosophy. They tend to split "artificial" from "intelligence", analyse both of them separately and then try to put them together in a sophisticated and holistic way. This second step almost never happens because the students - while in the first step deconstructing "artificial" - end in the void, concluding that the dichotomy artificial-natural makes no sense and that

\footnotetext{
* This work was supported by project "Interdisciplinary Partnership for Artificial Intelligence" (CZ.1.07/2.4.00/17.0055), OP Education for Competitiveness, funded by the European Social Fund in the Czech Republic and by the State Budget of the Czech Republic.

${ }^{1}$ In the words of John McCarthy, father of the term artificial intelligence, see http: / / www-formal. stanford.edu/jmc/whatisai/nodel.html, or cf. Chapter 3 of this volume.
} 
there is nothing like being artificial or being natural. This is fair enough - it can happen to any term of natural language as long as it is attacked so strongly and deconstructed so thoroughly - but what if our world simply asks for a conceptualisation that distinguishes such an important categorical difference which can be seen for example between a plastic bag and a polypore, between city streets and a forest, between a house and a cave, or between an airplane and a bumblebee.

Maybe the artificial-natural dichotomy is not inherent to the world itself. However, it is surely inherent to our world conceptualisation, and above all, maintaining this dichotomy is simply useful, at least much more useful than dismissing it and pretending we do not see the difference (albeit the ability to see the difference does not imply the ability to say the difference).

I will now try to be rather constructive than deconstructive in this chapter and I will offer a possible way of characterising and formalising the distinction between artificial and natural, keeping in mind the usefulness that it should bear. My definition of natural and artificial should be useful for the AI discourse and should help when speaking about the disappearing human-machine divide seen as crossing (in both directions) the border between natural and artificial.

\section{Human Naturalness}

It is not very common to refer to ancient Greek philosophical terms in an engineering publication but here I feel such a step might be fruitful. The two concepts that I would like to bring to attention are physis and logos. In classical philosophy, they usually even do not form the opposites, but contrasting them here makes sense.

Physis is simply nature or naturalness, it is a concept referring to all things that grow on its own, intrinsically, to things that are in and created by the nature. It was used by the Greek god Hermes when he pulled out a plant to show Odysseus its intrinsic way of growth. Logos, on the other hand, means the whole complex and metaphorical concept of speech, meaning, human reason, rationality.

Physis connotates with raw untamed things, growth, procreation, reactivity, wilderness, warmth, dynamics, spontaneity, vortex, chaos, vagueness, mud, dust, rot, worms, turmoil, and also excessive growth (as in cancer). Logos connotates with clarity, purity, intentionality, geometry, logic, cold, statics, copying, algorithm, and also excessive loss (as in Alzheimer's disease).

If we put physis on one side of a continuous, somehow intuitive spectrum, and logos on the opposite side then we should put human right in the middle of the spectrum. Metaphorically speaking, human is a being of tension between physis and logos, a being producing and produced by this tension, a being that possesses about the same from both realms. I will try to explain this in more detail later. For now, it is important to note that "being as natural as human" thus means "having the same ability to balance in the equilibrium between physis and logos". Therefore, we would expect also a strong AI to be like this. 
Every thing, every object has its share of artificial and natural. There is no object purely natural because the objectness itself is the first trace of artificialisation. Understanding a fragment of reality as an object is a matter of logos and thus it gives the first blow to its pure naturalness. Tools of logos pull out pieces of inherently non-structured physis and construct shapes and objects from them. When Hermes showed the herb, drawing it from the ground to demonstrate its nature, its physis, the physis was already retreating. It was still somehow very strongly there, much more strongly than if Hermes had shown a plastic bag or a transistor, but no more in its pure form because artificiality has already crept in - the herb "being shown" is not the herb "being natural" inseparably in its physis.

What Hermes did, was something very typical for human, or even delimiting for human mind, thought and intelligence. If it were an animal instead of a human, it would not show/objectify/name the herb - it would simply transparently share with the herb their "unspoiled" and non-conceptualised physis together. On the other hand, if it were a machine instead of a human, it would operate only with purely symbolical representations completely detached from the intrinsic substance of the herb, and the herb itself would be substituted by a single symbol, or a symbolic representation of its geometrical model, or a symbolic representation of its molecular structure, or something similar. In other words, logos dissolves physis, and human is - in his nature - a steersman constantly oscillating around this unstable equilibrium where objects appear from the mud of physis before they disappear in the void of logos. We can also see this metaphor as a keen connotation to Wiener's and Ashby's cybernetics.

We can say that a major tool for such steering is natural language. Language in general is a long bridge between physis and logos, with deixis and protolanguages close to the bank of physis, formal languages, mathematics, geometry etc. close to the bank of logos, and natural language somewhere in between, where human minds operate. Human naturalness is thus something significantly different from naturalness seen merely as physis - human naturalness is an indivisible and intrinsic combination of natural and artificial, continuously re-enacted by the process of life itself. Therefore, the goal of the research field of Artificial (General) Intelligence is not building physis from logos (that would most likely be impossible) but rather pulling these two realms together and strike a new conscious mind on their frontier - this is probably doable.

\section{$3 \quad$ Natural and Artificial Objects}

When we use language to further analyse a freshly objectified (shown) object in more detail, we go step by step over this bridge and we start losing more and more of the object's naturalness. The object in itself stays the same but we receive more and more detached abstract concepts. Although such concepts are new objects transferable by means of language, they lose their connections to the inherent givenness of the original object. For example, no human being can describe in words how the root of a herb (or a cloud, a bird's nest, a coral) is 
exactly. The moment closest to the root's naturalness is when we show it (deixis), and since then the more we say about it in an attempt to fully describe it, the more artificial construct we get. At some point, the length of the description reaches beyond the limit of any human being and becomes manageable only by symbol-processing machines, having no meaning for human while being in this logos domain. For example, a "sentence" with 10 million "words" might be quite a good description of how the root is, but only as long as we interpret it as a 10-megapixel photograph of the root, forgetting everything about the language and humbly returning back to showing the root or at least its image, i.e. back to much more physis-related deixis. The logos-based interpretation of those 10 million symbols (i.e. reading and understanding them one by one) has absolutely no meaning for us.

So what is it natural? Natural is that which defies being captured by language. Naturalness is everywhere where we feel tension between what we wanted to capture by our words and what we really captured. The more tension, the more naturalness we just encountered. Natural is something that we have to abstract away from in order to capture it by language.

On the other hand, artificial is imposed by language - artificial is that whose essence is fully determined by language. The artificial is a language abstraction drawn from the soil of physis and attracted by the clarity of logos.

Let's imagine an old rustic wooden table. What is artificial about it? That which we can grasp with words: shape and size of its geometrical idealisation, its weight, colour tone, purpose, or perhaps a description of the way it was made by a carpenter with an axe, a saw and a jack plane. However, we cannot describe how exactly it looks, how it feels when being touched, the exact look of its texture and wood structure, its smell.

Now let's imagine a three-legged white round plastic garden table. How to grasp it with words? Just take its designer's drawings and the description of technological aspects of its manufacturing and we have it right in front of us. We do not need to see and touch and feel this table to fully know how and what it really is - hence it is almost completely artificial. Yet even such an artificial thing has something natural about it: various scratches, defects, imperfections, shabbiness, but most importantly its inherent qualia potential that we exploit when we meet the table right here and now. All these aspects defy being captured by words, and therefore are natural.

Apart from what has been said above about the artificial, we can add that the artificial is the means of our language-supported understanding of the world. However, not much more can be said about the artificial itself - the more we say about it, the more we feel that we are loosing its original concept; on the meta-level, the concept of artificiality itself defies being captured by language. Therefore, the concept of artificiality is very much natural itself - and so the artificial is the natural means of our understanding of the world. 


\section{Emergence and Artificially Built Naturalness}

Through language, we can build a conceptualisation scaffolding around the world. We build it step by step, further and further. We know that if we build a floor of the scaffolding, we can add one more. Yet we know that we can never reach the sky; we can never breach the horizon - it would always become the chasing of a rainbow. ${ }^{2}$ But - at least we know everything about this logosoriginating scaffolding. We know everything about the world it encompasses, as much as we can know about a landscape from a map: the map is not for feasting one's eyes on the beautiful countryside, but for perfect orientation it is quite enough. The scaffolding itself is very much artificial and can be exemplified for example as a particular domain of a scientific discourse. Those things in the scaffolded world, for which "feasting one's eyes" equals "perfect orientation", are purely artificial. The rest is still more or less pertaining to naturalness especially the world beyond the horizon where the scaffolding does not reach.

However, what if we insist on building the scaffolding even beyond the horizon? We can construct a machine that will do it for us (just like in case of the aforementioned 10-megapixel photograph). The machine will pile up the scaffolding floors on top of each other so quickly that it will soon reach the sky and even further. But what is such a new scaffolding for us? We still stand where we were before and we know that we will never be able to climb up to the top to see how it looks beyond the horizon. The scaffolding itself thus ceases to be lucid for us anymore and starts to defy being captured by language. Physis strikes back. Physis again finds its way to the part of the world from which it was expelled.

In other words, when complexity of artificially built systems reaches a level on which it becomes impossible to describe them in finite ${ }^{3}$ time - to capture them by language - then the wild and chaotic world takes back what belongs to it anyway and those systems start to become natural. Maybe not at once, but naturalness gradually starts to proliferate through them.

This is exactly the trick of emergentism and emergent phenomena. All we need is quantity. Quantity beyond the horizon. A system may consist of purely artificial, perfectly describable, human-made elements. One such an element can be captured by language. Two of them as well. Three, four, five, ... still can be captured by language, hence still artificial. However, if the system consists of 100 billion such mutually interacting elements, it definitely cannot be captured by language - perhaps it can be captured by that super-high scaffolding, but such a scaffolding cannot be captured itself, so it makes no difference. It is just like in sorites, "little-by-little" paradoxes - only there is nothing paradoxical about it; it is simply the phenomenological givenness of how we perceive the world. Physis thus comes back to the system, no matter the artificial in its elements. To put it simply: emergent phenomena are natural, not artificial.

\footnotetext{
${ }^{2}$ This is actually a rather poetic, informal and intuitive ultra-short introduction to natural infinity, one of the key concepts of Vopěnka's Alternative Set Theory [1, 2].

${ }^{3}$ Here 'finite' in the non-standard sense of Vopěnka's Alternative Set Theory, i.e. the opposite of naturally infinite.
} 
If Artificial Intelligence (now we mean it as a "scientific discipline") creates an "artificial" mind emerging on top of an immensely complex system, this mind will be natural! As natural as our minds are. However, it will not be the AI engineers who are the authors or creators of its naturalness, who shall take the credit for it. The naturalness will be given to it from the same source and by the same means as it is given to everything else in the world. The AI engineers only prepare a substrate for it and then try to build the scaffolding high enough to lure the emergence through it.

AI research and development is metaphorically a Kabbalistic practice of its kind. A group of more or less wise men mould very complex inanimate matter, following strong rules, rituals and traditions, and then they ritually dance around this matter and heap up myriads of words arranged into very sophisticated spells, hoping that these words will evoke the spirit of emergence which brings naturalness and life into the artificial and inanimate.

This is the reason why GOFAI - Good Old-Fashioned Artificial Intelligence, i.e. "classical" AI in its symbolic, top-down paradigm [3] - has not achieved to create anything natural. In GOFAI, the AI engineer is also The Creator, the one who knows how the system works and what it is that makes it intelligent, thinking, with mind. Therefore, the whole system is in front of the horizon, fully within the lucid structure of the scaffolding built by the engineer, fully captured by language - hence fully artificial. A man can be a creator, but only of the artificial.

Emergent AI is in a very different situation: naturalness leaks into artificially created systems through their immense complexity that lies far beyond the horizon of what can be captured by language. However, the AI engineer has a fundamentally different role here: he is not The Creator anymore, and he remains only a priest, sage, shaman, theurgist. He knows what he did but he does not know what exactly it is that makes the system intelligent, aware, sentient, thinking.

So what are our Artificial Intelligence dreams about? If they are about us being The Creators of new natural artificial intelligence and minds, then we really dream Artificial Dreams. Yet it is natural to dream Artificial Dreams, and perhaps even pleasant, comforting and helpful. But when we wake up from the dreams, we should seriously start to think how to live with the natural machine intelligence that has already started to emerge on top of our technological artefacts. The disappearing of the human-machine divide - as Kevin Warwick in the first chapter of this book offers - is now much less surprising when we realise that the distance between human and machine is countless times smaller than between physis and logos.

\section{References}

1. Vopěnka, P.: Mathematics in the Alternative Set Theory. Teubner, Leipzig (1979)

2. Vopěnka, P.: The Great Illusion of 20th Century Mathematics and Its New Foundations. Preprint. University of West Bohemia, Pilsen (2012)

3. Haugeland, J.: Artificial Intelligence: The Very Idea. MIT Press, Cambridge (1985) 